\title{
DEVELOPMENTS OF POLYHEDRA USING OBLIQUE COORDINATES
}

\author{
J. AkiYAma AND C. NARA
}

\begin{abstract}
This paper is an exposition of the results in the paper entitled "Convex Development of a Regular Tetrahedron" by J. Akiyama, et. al. [1]. We fill in the detail which have been omitted in the paper. We determine all convex developments of a regular tetrahedron $V$ using a tiling generated by $V$ and we arrive at conditions for convex polygons to be convex develpments of $V$. Moreover, we identify all convex polyhedra whose convex developments can be determined by the method used for $V$.
\end{abstract}

\section{INTRODUCTION}

A development of a polyhedron is a plane figure obtained by cutting the surface of the polyhedron. Cuts need not be confined to edges but can also be made along faces (Figure 1). Hence a polyhedron has infinitely many developments. It is showed in [1] that we can systematically get infinitely many convex developments of a regular tetrahedron using a tiling. The similar results for a doubly-covered square are shown in [2] and ones for flat 2-foldings of a convex polygons are in [3]. This paper is an exposition of based on [1]. We fill in the details which have been omitted and provide expanded versions of the proofs.

Figure 1:

Received 17 January 2006, revised 12 June 2006, accepted 4 July 2006. 2000 Mathematics Subject Classification: 52

Key words and Phrases:tetrahedron, development, tiling, convexity 
We consider only straight line cuts. The main goal is to determine all convex developments of a regular tetrahedron using a tiling which is described in Section 2.2. In Section 2.1, we give a simple proof to show that there is no convex $n$-gon which is a development of a regular tetrahedron when $n \geq 7$ (Theorem 2 in [1]). Section 3 discusses the necessary conditions for a convex polygon to be a development of a regular tetrahedron. Sections 2.4, 2.5, 2.6 and 2.7 explain the necessary and sufficient conditions under which triangles, quadrilaterals, pentagons and hexagons, respectively, are convex developments of a regular tetrahedron. Section 2.8 shows how to determine all polyhedra whose convex developments can be obtained using the tiling method of Section 2.2. Section 2.9 discusses convex developments of $n$-folded polygons.

\section{MAIN RESULTS}

\subsection{Classification of Developments}

Let $\alpha$ be a development of $V$. Then each point in $\alpha$ corresponds to only one point in $V$. Let $P$ be a point in $\alpha$. If $P$ is a point in the interior of $\alpha$, then there is a circle in $\alpha$ with center at $P$, so we say the angle at $P$ in $\alpha$ is $2 \pi$. If $P$ is a point on the boundary of $\alpha$, but is not a vertex of $\alpha$, there is a semi-circle in $\alpha$ with center at $P$, so we say the angle at $P$ in $\alpha$ is $\pi$.

If $P$ corresponds to a vertex $Q$ in $V$, then the sum of the three angles formed by the faces of $V$ that meet at $Q$ is $\pi$. Hence $P$ must be a point on the boundary of $\alpha$. If $P$ is a point on the boundary of $\alpha$ but is not a vertex of $\alpha$, there is no point in $\alpha$, other than $P$, which corresponds to the vertex $Q$ since the angle at $P$ in $\alpha$ is $\pi$.

Property 2.1. In a convex development $\alpha$ of $V$, all vertices of $V$ correspond to points on the boundary of $\alpha$. Moreover, a vertex $P$ of $V$ corresponds to only one point in $\alpha$, unless it corresponds to a vertex of $\alpha$.

Since the development is convex, vertices in must satisfy the following condition.

Property 2.2. The angle of a vertex in a development is less than $\pi$.

Property 2.3. [1] Let $\alpha$ be a convex development of a regular tetrahedron and let $P$ be a point in $\alpha$. Let $\theta$ be the angle at $P$ in $\alpha$, then $\theta<\pi$ if $P$ is a vertex of $\alpha$, $\theta=\pi$ if $P$ is interior to an edge of $\alpha$, and $\theta=2 \pi$ if $P$ is a point in the interior of $\alpha$.

Fold a development $\alpha$ to the regular tetrahedron $V$. Then a point $P$ in $\alpha$ corresponds to either a vertex of $V$, a point interior to an edge of $V$, or a point in 
the interior of a face of $V$. More specifically, vertices $P$ in $\alpha$ can be classified into three types as follows:

(1) $P$ corresponds to a vertex in $V$ and together with other vertices of $\alpha$ corresponding to the same vertex in $V$ forms an angle $\pi$ in $V$.

(2) $P$ corresponds to a point on an edge of $V$ and together with other vertices of $\alpha$ corresponding to the same point in $V$ forms either an angle $\pi$ or $2 \pi$ in $V$.

(3) $P$ corresponds to a point $Q$ in the interior of a face of $V$ and together with the other vertices in $\alpha$ corresponding to $Q$ forms either an angle $\pi$ or $2 \pi$ in $V$. Moreover, if the angle is $\pi$, then there is a point on the boundary of $\alpha$ which corresponds to the point $Q$ and whose angle in $\alpha$ is $\pi$.

Lemma 2.4. Let $\alpha$ be a convex n-gon which is a development of $V$. Then the average size of the angles of those vertices in $\alpha$, which contribute to the angle at a point in $V$, is less than or equal to $2 \pi / 3$.

Proof. By Property 2.3., if we use only vertices of $\alpha$ whose angles are less than $\pi$, we need at least two or three vertices to cover the angle $\pi$ or $2 \pi$. Hence the average size of angles of vertices of $\alpha$, which contribute to the angle at a point in $V$, is less than or equal to $2 \pi / 3$.

The proof of the following theorem uses Lemma 2.4..

Theorem 2.5. There are no convex n-gons which are developments of a regular tetrahedron when $n \geq 7$.

Proof. Since the sum of the interior angles of an $n$-gon is $(n-2) \pi$, by lemma 1 we have $(n-2) \pi \leq \frac{2}{3} \pi n$, that is, $n \leq 6$.

\subsection{Tiling Generated by a Regular Tetrahedron}

In this section we discuss the relationship between developments and tilings. Take a regular tetrahedron $V$ and carve distinct figures on each of its four faces, dip the faces in ink and print the figures on a blank sheet as follows: First, print the figure on one face, choose one edge of the face as an axis, rotate the tetrahedron along the axis and print the figure on the other face. Repeat the process continuously. The printed pattern that results is a periodic tiling of the plane. Moreover, the same pattern will result regardless of the direction or order in which the tetrahedron is rotated. Whenever such a tiling can be obtained from a polyhedron, we say that the polyhedron generates a tiling of the plane.

Among regular polyhedra the regular tetrahedron is the only one which can generate a tiling of the plane in the given way. This stems from the fact that for 
any vertex $P$ of a regular tetrahedron, there exists an integer $n$ such that if $T$ is the sum of the angles formed by the three faces of $V$ which meet at $P$, then $n T=2 \pi$. This is a necessary condition for a polygon to generate a tiling.

If we observe the trace of a point on the surface of $V$ and the features of the positions it occupies, we will be able to find conditions under which tilings exist, conditions which can be identified with properties of developments of regular tetrahedra.

It is convenient to use oblique coordinates on the plane $\gamma$ and to consider a development of $V$ as a figure on $\gamma$ (Figure 2). A point $P(x, y)$ of $\gamma$ is a lattice point if both $x$ and $y$ are integers.

Definition 2.6. Two points $P$ and $Q$ in the plane $\gamma$ are equivalent if either the mid-point of the segment between them is a lattice point or if both the differences between corresponding coordinates are even integers. The former is called symmetry type equivalence, while the latter is called parallel moving type equivalence.

For a set $S$ of points in the plane $\gamma$, we say that points in $S$ are mutually equivalent if any two points in $S$ are equivalent. Recall the tiling obtained from the distinct figures carved on the four faces of $V$. Any equivalent points in the plane $\gamma$ correspond to the same points on $V$, i.e., each point on $V$ corresponds to a set of points in the plane which are mutually equivalent. Note that if $P$ and $Q$ are equivalent and if $Q$ and $R$ are equivalent, then $P$ and $R$ are also equivalent, so the relation mentioned above is an equivalence relation. All points in $\gamma$ can be classified into equivalence classes with respect to this equivalence relation.

Note also that all four vertices of the regular tetrahedron $V$ correspond to lattice points in the plane $\gamma$. Let $S$ be a subset of lattice points in which no two are equivalent. Then $S$ has at most four points since every lattice point in the plane $\gamma$ corresponds to one of four vertices of $V$. If all points in $S$ are on the same line in the plane $\gamma$, then $S$ has at most two points because of the definition of equivalence. Given three lattice points $P, Q$ and $R$ in the plane $\gamma$, the point $X$ which completes the parallelogram $P Q R X$ is also a lattice point.

Theorem 2.7. A development of a regular tetrahedron $V$ is a closed connected subset $S$ of the plane $\gamma$ containing representatives from all equivalence classes in $\gamma$ and no two points in its interior are equivalent. 
Figure 2: Points with the same marks are mutually equivalent

\subsection{Basic Properties}

For the remainder of the paper, a development of a regular tetrahedron $V$ refers to a development of $V$ described in the plane $\gamma$. We call the triangle $\triangle E F G$ in Figure $3 a$, a basic development of a regular tetrahedron $V$. After cutting off a part $S$ of a basic development, rotate it by the angle $\pi$ about a lattice point, or shift it by an even number of units parallel along one of the axes. Many distinct developments of $V$ (Figure $3 b, 3 c, 3 d, 3 e, 3 f$ ) can be obtained by repeating this procedure.

Denote the four vertices of $V$ by $A, B, C$ and $D$. We will use the same letters $A, B, C$ and $D$ for the lattice points in the plane corresponding to vertices of $V$ for the remainder of the paper. Then no two points called $A, B, C$ or $D$ in $\gamma$ are equivalent. In general, letters $E, F, G, H$, etc. will denote vertices of developments.

Property 2.8. Let $\alpha$ be a development of $V$ in the plane $\gamma$ and let $E F$ be a side of $\alpha$. Then there are at most three lattice points on EF. If there are exactly three lattice points on $E F$, then $E$ and $F$ are equivalent.

Proof. Suppose that there are three lattice points $P, Q$ and $R$ on the side $E F$. 
Since at most two lattice points on a line are not equivalent, then two of $P, Q$ and $R$, say $P$ and $Q$, must be equivalent. Hence both $P$ and $Q$ correspond to the same vertex of $V$, and so the sum of the angles at $P$ and $Q$ in $\alpha$ is at most $\pi$. Thus $P$ and $Q$ are endpoints on a side in $\alpha$, that is, $P Q=E F$ and $E$ and $F$ are equivalent.

We can assume without loss of generality that a basic triangle has area 2 , therefore the smallest triangle with vertices at lattice points has area $1 / 2$. Since Pick's theorem holds in the plane $\gamma$, the area of a polygon whose vertices are lattice points is given as follows: (the number of lattice points on the boundary) $/ 2+$ (the number of lattice points inside of the polygon).

Property 2.9. Let $\alpha$ be a convex development of a regular tetrahedron $V$. There are four lattice points on the boundary of $\alpha$, corresponding to $A, B, C$ and $D$, which make a parallelogram with area 1.

Proof.

(1) $\alpha$ contains four lattice points $A, B, C$ and $D$ which correspond to four distinct vertices of $V$, and by the property 1 they are on the boundary of $\alpha$.

(2) We will show that a convex quadrilateral $A B C D$ (if necessary, exchange the letters $A, B, C, D)$ is a parallelogram. Suppose that the quadrilateral $A B C D$ is not a parallelogram. Since the quadrilateral $A B C D$ is convex, we can choose three points among four points $A, B, C$ and $D$ which make a parallelogram with another point $X$ in $\alpha$. Since three points of the parallelogram are lattice points, $X$ is also a lattice point. Hence $X$ should be on the boundary of the quadrilateral $A B C D$, say on $A B$, by Property 1 . Now there are three lattice points $A, X$ and $B$ on the same edge in this order. By property $4, A$ and $B$ are equivalent, which contradicts the fact that $A$ and $B$ come from distinct vertices of $V$. Thus the quadrilateral $A B C D$ is a parallelogram.

(3) Since the quadrilateral $A B C D$ does not include any lattice point in its interior as well as on its boundary except for $A, B, C$ and $D$, its area is 1 by Pick's theorem.

Property 2.10. Let EF and $F G$ be adjacent sides of a development $\alpha$ of a regular tetrahedron $V$ such that $E, F$ and $G$ are mutually equivalent.

(1) If $E$ and $F$, as well as $F$ and $G$, are parallel moving type equivalent, then $\triangle E F G$ is a development $\alpha$.

(2) If $E, F$ and $G$ are not lattice points, then at most one of the two edges EF and $F G$ can contain two lattice points.

Proof.

(1) Suppose that $E$ and $F$, as well as $F$ and $G$, are parallel moving type equivalent. Let $\triangle E^{\prime} F^{\prime} G^{\prime}$ be a triangle obtained by moving $\triangle E F G$ parallel to the axes such that $F^{\prime}$ is a lattice point. Since $E$ and $F$, as well as $F$ and $G$, are parallel moving type equivalent, $E^{\prime}$ and $G^{\prime}$ are also lattice points. The area of $\triangle E^{\prime} F^{\prime} G^{\prime}$ is two, hence the area of $\triangle E F G$ is two. Therefore $\triangle E F G$ itself is a development $\alpha$. 
(2) Suppose that there are two lattice points $P, Q$ on the edge $E F$ and two lattice points $R, S$ on the edge $F G$. Then the quadrilateral $P Q R S$ is convex but not a parallelogram. Hence there is a lattice point in the interior of $\alpha$, which contradicts property 1. Thus it is impossible that both $E F$ and $F G$ have two lattice points each unless $E, F$ and $G$ are all lattice points.

The following are the basic conditions for a closed connected subset $\alpha$ of the plane $\gamma$ to be a development of $V$ :

(1) There are no lattice points in the interior of $\alpha$.

(2) There are four lattice points $A, B, C$ and $D$ on the boundary of $\alpha$, no two of which are equivalent, and they form a parallelogram with the area one.

(3) The area of $\alpha$ is two.

\subsection{Triangles}

In this section, discuss the conditions for a triangle to be a development of $V$.

Theorem 2.11. If a development of $V$ is a triangle $\triangle E F G$ in the plane $\gamma$, then $E, F$ and $G$ are mutually equivalent, and the development satisfies the basic conditions. Conversely, if a triangle in the plane $\gamma$ satisfies the basic conditions and if $E, F$ and $G$ are mutually equivalent, then $\triangle E F G$ is a development of $V$.

Proof. Let $\triangle E F G$ be a development $\alpha$ of $V$. Since the sum of the interior angles of $\triangle E F G$ is $\pi$, three points $E, F$ and $G$ come from the same point of $V$. Hence $E, F$ and $G$ are mutually equivalent.

Proof of the converse. Let $\triangle E F G$ be a triangle with area 2 in the plane $\gamma$. Suppose that $E, F$ and $G$ are mutually equivalent and that $\triangle E F G$ satisfies all the basic conditions. Then either all three points $E, F$ and $G$ are lattice points or none of them is a lattice point. 
(1) Suppose that $E, F$ and $G$ are lattice points. Since they are mutually equivalent, the mid-points of $E F, F G$ and $G E$ are lattice points. By Theorem 2, $\triangle E F G$ is a development of $V$.

(2) Suppose that none of $E, F$ and $G$ is a lattice point. Two of four points $A, B, C$ and $D$ are on the same side, say on $E F$. By property 4 , there are at most two lattice points interior to a side. Hence $E$ and $F$ are parallel moving type equivalent. One of the other two lattice points is on $F G$ and the other is on $G E$ by the property $6(2)$. Since $E, F$ and $G$ are mutually equivalent, those lattice points are the mid-points of $F G, G E$ (Figure $4 b$, Figure $4 c$ ). Thus $\triangle E F G$ is a development of $V$.

Corollary 2.12. A right isosceles triangle cannot be a development of a regular tetrahedron $V$.

Proof. Suppose that $\triangle E F G$ is a right isosceles triangle which is a development of $V$. Then the three points $E, F$ and $G$ are mutually equivalent by Theorem 3 , and at least two of the mid-points of $E F, F G$ and $G E$ are lattice points by the property $6(1)$. Denote the length of an edge in $V$ by $a$. Then the distances between pairs of lattice points, are either $a$ or greater than or equal to $\sqrt{3} a$. Each case contradicts the fact that the area of a development is $\sqrt{3} a^{2}$. This completes the proof of the corollary.

\subsection{Quadrilaterals}

In this section, we discuss criteria for a convex quadrilateral to be a development of $\mathrm{V}$.

Theorem 2.13. If a development of $V$ is a convex quadrilaterala in the plane $\gamma$, then it satisfies one of the following conditions:

(1) All mid-points of sides are lattice points (Figure 5a). (2) $\alpha$ is a parallelogram and the endpoints of each side of a pair of parallel sides are equivalent (Figure $5 b$ ). (3) $\alpha$ is a trapezoid which is not a parallelogram, the endpoints of each of its two legs are equivalent, and at least one of the mid-points of its two legs is a lattice point.

Conversely, if a convex quadrilateral in the plane $\gamma$ satisfies the basic conditions and if it satisfies one of the above conditions (1), (2),(3), then it is a development of $V$.

Proof. Let a quadrilateral $E F G H$ be a development of $V$. Since the sum of the interior angles of the quadrilateral is $2 \pi$, by property 3 there are two possibilities:

Case 1: All four vertices $E, F, G$ and $H$ correspond to one point, denoted by $P$ in the interior of a face of $V$. Then four vertices $E, F, G$ and $H$ are mutually equivalent and they are not lattice points. 
We will show that each side has exactly one of four lattice points $A, B, C$ and $D$ corresponding to the vertices of $V$. Suppose that this does not hold. Then there are two lattice points, say $A$ and $B$, on the same edge, say $E F$, by property 1 . Since $E$ and $F$ are parallel moving type equivalent by property 4 , the mid-points of each of the edges $F G$ and $H E$ are lattice points corresponding to $C$ and $D$, respectively, by the property $6(1)$. The length of the edge $E F$ is twice that of the edge $A B$, which leads to the contradiction $G=H$. Therefore, the four vertices $A, B, C$ and $D$ are on distinct edges of the quadrilateral EFGH. Furthermore, they are the mid-points of the edges of the quadrilateral since the endpoints of each edge are equivalent (Figure 4a).

Case 2: The four vertices $E, F, G$ and $H$ are divided into two pairs, say $E$ and $F, G$ and $H$, such that the sum of the interior angles for each pair is $\pi$ and the points in each pair come from the same point in $V$. Hence the edges adjacent to the edge $E F$ are parallel to each other. Thus the quadrilateral is a trapezoid with the following possibilities:

(i) If $E F$ and $G H$ are parallel to each other, the quadrilateral is a parallelogram (Figure $5 b$ ).

(ii) If $E F$ and $G H$ are not parallel to each other, then at least one of the mid-points of $E F$ and $G H$ is a lattice point; for if $E, F$ and $G, H$ are parallel moving type equivalent, the intersection of a line passing through $E$ and parallel to $G H$, with the edge $F G$, makes a triangle $E F P$ whose area is two, which is a contradiction.

Figure 5d

Proof of the converse. Let EFGH be a convex quadrilateral in the plane $\gamma$ which 
satisfies the basic conditions and satisfies one of the conditions (1), (2) or (3) in theorem 4 . We divide the proof into three cases as follows:

(1) The mid-points of all the edges of the quadrilateral are lattice points. Denote them by $A, B, C$ and $D$. Since the area of the parallelogram $A B C D$ is $1, E F G H$ is a development of $V$ by Theorem 2 (Figure $5 a$ ).

(2) $E F G H$ is a parallelogram and $E$ and $F$, as well as $G$ and $H$, are equivalent. Since the area of $E F G H$ is 2, for lattice points corresponding to $A, B, C$ and $D$, either a pair of parallel edges of $E F G H$ each have two lattice points, or each of the four edges has one. Then either case satisfies the conditions in Theorem 2, and hence $E F G H$ is a development of $V$.

(3) $E F G H$ is a trapezoid but not a parallelogram, say $E H$ and $F G$, are parallel to each other but $E F$ and $G H$ are not parallel to each other.

By condition (3) in the statement of Theorem 4, we can assume without loss of generality that the mid-point of $E F$ is a lattice point (denoted by $A$ ). Draw a line $l$ parallel to $G H$ passing through $A$. Let $E^{\prime}$ and $F^{\prime}$ respectively be intersections of $l$ with the lines $E H$ and $F G$, respectively. Then the parallelogram $E^{\prime} F^{\prime} G H$ is a development of $V$ by the proof of case (2). Thus $E F G H$ is also a development of $V$ (Figure 5e), since each point in $\triangle E^{\prime} A E$ corresponds to a point in $\triangle F^{\prime} A F$ which is symmetric type equivalent to it. This completes the proof of Theorem 4 .

Figure 5e

Corollary 2.14. A square cannot be a development of a regular tetrahedron $V$.

Proof. Suppose that a square $E F G H$ is a development of $V$. Then either all mid-points of edges of $E F G H$ are lattice points (denoted by $A, B, C$ and $D$ ), or the parallel edges, say $E F$ and $G H$, have two each of the four lattice points. The former case contradicts the fact that the distance between any two lattice points is equal to the length (denoted by $a$ ) of an edge of $V$ or is greater than or equal to $\sqrt{3} a$. The latter case contradicts the fact that the area of developments of $V$ is 2 .

\subsection{Pentagons}


In this section, we present criteria for a pentagon to be a development of $V$.

Theorem 2.15. If a development of $V$ is a convex pentagon, denoted by EFGHI, then the development satisfies the basic properties and, in addition, it satisfies the following condition $(*)$ :

$\left(^{*}\right)$ : The mid-points of the edges $E F$ and $F G$ are lattice points, $G H$ and $E I$ are parallel to each other, and $H$ and $I$ are equivalent (Figure 6), where EF and $F G$ are any two adjacent edges of the pentagon.

Conversely, if a convex pentagon in the plane $\gamma$ satisfies the basic conditions and condition $(*)$, then the pentagon EFGHI is a development of the regular tetrahedron $V$.

Proof. Let a convex pentagon EFGHI be a development of $V$. Since the sum of the interior angles of the pentagon is $3 \pi$, then by property (3) there is only one possible combination of five vertices so that the sum of interior angles of three of them, say $\angle E, \angle F$ and $\angle G$, is and that the sum of the interior angles of the other two, $\angle H$ and $\angle I$, is $\pi$. Hence the following statements hold:

(1) Three vertices $E, F$ and $G$ are mutually equivalent. They are not lattice points; otherwise, the mid-points of each of the three edges are also lattice points and hence the area of $\angle E F G$ is 2 , which contradicts the fact that the area of the development $E F G H I$ is 2.

(2) Two vertices $H$ and $I$ are equivalent and two edges $G H$ and $E I$ are parallel to each other by corresponding angles.

(3) At least one of the mid-points of the two edges $E F$ and $F G$ is a lattice point (denoted by $A$ ) by property 5(1). Suppose that the mid-point of the edge $E F$ is a lattice point but the mid-point of the edge $F G$ is not. Then $F$ and $G$ are parallel moving type equivalent. Draw an edge $A P$ parallel to the edge $F G$ assing through $A$ with the same length as $F G$. Then the edge $A P$ is in the convex pentagon $E F G H I$. Since $A$ and $P$ are parallel moving type equivalent, $P$ is also a lattice point. Moreover, the mid-point of the edge $A P$ is a lattice point in the interior of the pentagon $E F G H I$, which contradicts property 1. Hence the mid-points of two edges $E F$ and $F G$ are lattice points. 
Proof of the converse. Let a convex pentagon EFGHI satisfy the basic conditions, as well as condition $(*)$. Then the mid-points of each of the two edges $E F$ and $F G$ are lattice points (denoted by $A$ and $B$ ) and the other lattice points $C$ and $D$ are on the edges $G H, H I$ or $I E$. If the lattice point $C$ is interior to the edge $G H$ (or $I E)$, the point $P$ which makes a parallelogram with the three points $A, B$ and $C$ is also a lattice point and it is in the pentagon $E F G H I$. By the basic conditions, $P$ is on the boundary of the pentagon $E F G H I$ and hence on the edge $H I$ (Figure $6 b$ ). If both $G H$ and $I E$ do not have lattice points interior to the pentagon $E F G H I$, then the edge $H I$ contains at least two lattice points (Figure $6 a$ ). Therefore, the pentagon $E F G H I$ is a development of $V$ by Theorem 2 .

\subsection{Hexagons}

In this section, we present criteria for a hexagon to be a development of $\mathrm{V}$.

Theorem 2.16. If a development of $V$ is a convex hexagon, denoted by EFGHIJ, then it satisfies the basic conditions and, in addition, it satisfies the following condition (**).

$\left.{ }^{* *}\right)$ : The mid-points of each of the four edges EF,FG,HI and IJ are lattice points, $A, B, C$ and $D$, no two of which are equivalent and the quadrilateral $A B C D$ is a parallelogram with area 1 .

Conversely, if a convex pentagon EFGHIJ in the plane satisfies the basic conditions and condition (**), then the pentagon EFGHIJ is a development of the regular tetrahedron $V$.

Proof. Let a convex hexagon EFGHIJ be a development of $V$. Since the sum of the interior angles of the pentagon is $4 \pi$, then by property (3) the six vertices are divided into two groups, say $E, F G$ and $H, I, J$, so that the sum of the interior angles of each group is $2 \pi$. Then three points $E, F$ and $G$ correspond to the same point $P$ in $V$ and hence are mutually equivalent. The point $P$ in $V$ is not a lattice point because the angle at $P$ in $V$ is $2 \pi$. By the property $6(1)$, at least one of the mid-points of the two edges $E F$ and $F H$ (similarly $H I$ and $I J$ ) is a lattice point. By reasoning in the manner shown in Figure $6 c$, none of the endpoints of 
Figure 7: Points with the same marks are mutually equivalent

the four edges $E F, F G, H I$ and $I J$ are parallel moving type equivalent. Thus the mid-points of all four edges $E F, F G, H I$ and $I J$ are lattice points corresponding to $A, B, C$ and $D$. $A B C D$ is a parallelogram with area 1 since there is no lattice point in the interior of the hexagon.

Proof of the converse. Let the convex hexagon EFGHIJ satisfy the basic conditions and condition $\left({ }^{* *}\right)$. Then the pentagon EFGHIJ satisfies the conditions in Theorem 2. Hence it is a development of $V$.

\subsection{Polyhedra which Generate Tilings}

A dihedron is a doubly covered polygon, i.e. it consists of two congruent faces joined along an edge. The two faces are related by a reflection. Call the shape of one face of a dihedron its face shape. A development of a dihedron is a plane figure obtained by cutting the surface of the dihedron.

The method in Section 2, which was used to determine if a convex figure in the plane $\gamma$ is a development of a regular tetrahedron, can be applied to other polyhedra. In this section we determine all polyhedra and dihedra which generate tilings of the plane.

Lemma 2.17 If the face shape of a dihedron $Q$ is a rectangle, an equilateral triangle, an isosceles right triangle or a right triangle with the angle $\pi / 3$, then the dihedron generates a tiling of the plane. Conversely, if a dihedron generates a tiling of the plane, then the face shape of the dihedron is one of the triangles mentioned above.

Proof. It is easy to check that a dihedron with a face shape which is a rectangle, an equilateral triangle, an isosceles right triangle or a right triangle with the angle $\pi / 3$, generates a tiling of the plane.

Conversely, if a dihedron generates a tiling of the plane, we will show that its face shape $Q$ is one of the four triangles mentioned in Lemma 2. Since the tiling has no gaps, the face shape of $Q$ is convex. Let the face shape of $Q$ be a $k$-gon $P_{1} P_{2} \ldots P_{k}$. Let $P$ be one of $P_{i}(i=1,2, \ldots, k)$. Denote the angle at $P$ by $\theta$. Then the sum of the angles at $P$ on the top and bottom faces $Q$ is $2 \theta$. Hence $2 \pi$ is an integer multiple of $2 \theta$. Let $2 n \theta=2 \pi$, that is, $\theta=\pi / n$. Then $n \geq 2$ since the face shape of the dihedron is convex. Hence $\theta \leq \pi / 2$. The sum of the interior angles of a $k$-gon is $(k-2) \pi$. Hence $(k-2) \pi \leq k \pi / 2$, that is, $k \leq 4$. 
Suppose $k=4$. The inequality is satisfied only when $\theta=\pi / 2$ for any point $P_{i}(i=1,2, \ldots, k)$. Thus the face shape is a rectangle.

Suppose $k=3$. The face shape is a triangle. Denote the three angles by $x, y$ and $z(x \geq y \geq z)$. Then $x+y+z=\pi$ and there are integers $l, m$ and $n$ greater than one such that $x=\pi / l, y=\pi / m, z=\pi / n$ (for some natural numbers $l, m, n$ ). Substituting these values in $x+y+z=\pi$, we get $\frac{1}{l}+\frac{1}{m}+\frac{1}{n}=1$. Since $x \geq y \geq z$, we have $\frac{1}{l} \geq \frac{1}{3}$, that is, $l \leq 3$. Therefore $l=2,3$.

In the case $l=2$, since $\frac{1}{m}+\frac{1}{n}=1$ and $m \leq n$, we have $m \leq 4$. Thus, $l=$ $2, m=3, n=6$ or $l=2, m=4, n=4$. Then the face shape is a right triangle with the angle $\pi / 3$ or an isosceles right triangle.

In the case $l=3$, we have $m=n=3$. Then the face shape is an equilateral triangle. This completes a proof.

Fold any acute triangle $E F G$ along its medians as shown in Figure 8 to obtain a tetrahedron. This tetrahedron will generate a tiling of the plane. Hence there are infinitely many non-congruent polyhedra that generate tilings of the plane. It is known that the tetrahedra with congruent acute triangular faces are the only polyhedra which generate tilings of the plane.

Figure 8:

Theorem 2.18. Let $Q$ be a convex polyhedron or a dihedron. If $Q$ generates a tiling of the plane, then $Q$ is a tetrahedron with congruent acute triangular faces or a dihedron whose face shape is a rectangle, an equilateral triangle, an isosceles right triangle or a right triangle with the angle $\pi / 3$.

\subsection{Developments of Flat $n$-foldings of a square.}

A polygon $P$ is called a flat $n$-folding of a polygon $Q$ if $Q$ can be folded to exactly cover the surface of $P n$ times, with no part of the surface of $Q$ left over. All convex developments of dihedra whose face shapes are convex polygons (including squares) are determined in [2] and [3]. In this section, we describe a procedure which can determine infinitely many distinct developments of a 4 -folding of a square.

Figure $9 a$ shows a 4 -folding of a square and Figure $9 b$ shows a development of this 4 -folding. The letter $\mathrm{F}$ in Figure $9 b$ suggests the hills and valleys of the 4 -folding. Figure $10 a$ shows another 4 -folding of a square while Figure $10 b$ shows a development of this 4 -folding. Again the images of the letter $F$ suggest the hills and valleys of the 4 -folding. 
A periodic tiling of the plane can be obtained by repeated mirror reflections of either of the two developments (Figure 11) given above. It is convenient to think of developments of a 4 -folding of a square as lying in the plane $\tau$ with the orthogonal coordinates.

Figure 11:

Two points $P(a, b)$ and $Q(c, d)$ in the plane $\tau$ are equivalent if either the mid-point of the segment between them is a lattice point or if both the differences between corresponding coordinates are even integers. This relation is an equivalence relation.

Figure 12:

Let $\alpha$ be a convex development of a 4 -folding of a square in the plane $\tau$. After cutting off a part of the development shown in Figure $9 a$, rotate it by the angle $\pi$ about a lattice point, or shift it by an even number of units parallel along one of the axes. Repeat this procedure to obtain infinitely distinct developments of a 4-folding of a square, some of which are shown in Figure 12. 


\section{FUTHER RESEARCH}

The problem of determining all convex polygons which are a development of a $n$-folding for $n \geq 2$ remains open. A more specific question is the following. Are there any convex polygons other than the rectangle which are developments of a $n$-folding for any $n$ ?

A regular tetrahedron is the unique polyhedron among regular polyhedra, which has a convex development. It is interesting to determine all convex polyhedra which have at least one convex development.

Acknowledgement. The authors would like to express their special thanks to Gisaku Nakamura who introduced this topic and its interesting facts to them. They also thank Mari-Jo Ruiz for her assistance in the preparation of this paper.

\section{REFERENCES}

1. J. Akiyama,K. Hirata, M. Kobayashi,and G. Nakamura, , "Convex Development of a Regular Tetrahedron", Computational Geometry: Theory and Applications 34 (2005), 2-10.

2. J. Akiyama And K. Hirata, "On Convex Developments of a Doubly-Covered Square", Combinatorial Geometry and Graph Theory (IJCCJT2003), Springer-Verlag LNCS 3330 (2004), 1-13.

3. J. Akiyama,K. Hirata, M. P. Ruiz, and J. Urrutia , "Flat 2-Foldings of Convex Polygons", Combinatorial Geometry and Graph Theory (IJCCJT2003), SpringerVerlag LNCS 3330 (2004), 14-24.

J. AKIYAMA: The Research Institute of the Educational Development, Tokai University, 2-28-4 Tomigaya, Shibuya, Tokyo 151-8677, Japan.

E-mail: fwjb5117@mb.infoweb.ne.jp

C. NARA: Department of General Education, Kyushu Tokai Univershity, Kawayo, MinamiAso, Aso, Kumamoto, 869-1404, Japan.

E-mail: cnara@ktmail.ktokai-u.ac.jp 